\title{
ell guminiguada
}

ISSNe: 2386-3374, pp. 41-63

\section{ROMPER EL CICLO DE LA INVISIBILIDAD. EL EMPLEO COMO VÍA PARA FAVORECER LA INCLUSIÓN}

Teresa Fontán Montesinos

Universidad de Las Palmas de Gran Canaria

Gisela Rivero Marrero

Asociación de Discapacitados Melody

Fecha de aceptación: 20 de diciembre de 2013

\section{RESUMEN}

En España las posibilidades de empleo de las personas en riesgo de exclusión social no son satisfactorias. Se trata de un problema que centramos en las concepciones educativas de la enseñanza-aprendizaje inicial, que orientan minoritariamente a la formación para el empleo como a la oferta y demanda de trabajo de esos colectivos. En nuestro sistema educativo de la desatención y marginación del alumnado en riesgo de exclusión social se pasó a la educación especial; luego, a la normalización e integración, lo que condujo a la inclusión educativa. En ese momento final que nos encontramos aparecen visiones innovadoras como la escuela para todos, empleo con apoyo, y vida con apoyo que desarrollan el paradigma de la autonomía y vida independiente. Conscientes de las luces y sombras del proceso de la educación inclusiva alertamos del peligro de la derogación de lo conseguido hasta ahora, y la defendemos como la única educación moralmente deseable para la integración social y laboral de los colectivos en riesgo de exclusión social.

Palabras clave: educación inclusiva, colectivos en riesgo de exclusión social, barreras para el aprendizaje y la participación, formación para el empleo.

\section{ABSTRACT}

Employment chances for people in risk of social exclusion are not satisfactory in Spain. This has been a problem at the core of educational conceptions concerning primary education, scarcely orientated towards the employment of such groups. In our system, from margination and neglect of our pupils in risk of social exclusion, we sent them to special education first, then to normalization and integration and finally 
Teresa Fontán Montesinos, Gisela Rivero Marrero

Romper el ciclo de la invisibilidad. El empleo como vía para favorecer la inclusión

to inclusive education. Nowadays, innovative visions such as school for all, employment and life with support are developing the model of autonomy and independent life.

Being conscious of the difficulties of the inclusive education process we alert here of the danger of revoking what has been achieved so far and endorse it as the only desirable moral education for labour and social integration of these groups.

Key words: inclusive education, groups in risk of social exclusion, learning and participant barriers, education for employment.

\section{UN POCO DE HISTORIA LEGAL Y SOCIAL DE LA EDUCACIÓN INCLUSIVA EN ESPAÑA}

En el sistema educativo español la educación de las personas con discapacidad ha evolucionado muy favorablemente. De la desatención y marginación iniciales se pasó a la educación especial, luego a la normalización e integración, lo que condujo a la inclusión educativa. En ese momento final en el que nos encontramos aparecen visiones innovadoras como las de la escuela para todos, empleo con apoyo, y vida con apoyo que acaban desarrollando el paradigma de la autonomía y vida independiente que defenderá la incorporación de las personas con discapacidad a la vida social y al empleo a través de la formación como fortalecimiento o capacitación (empowerment) ${ }^{1}$.

\subsection{Precedentes de la educación inclusiva: los años 70}

Por primera vez el sistema educativo español se plantea legalmente la educación de los alumnos con algún tipo de discapacidad en la Ley 14/1970, de 4 de agosto, General de Educación y Financiamiento de la Reforma educativa (en adelante, LGE). La LGE señala, en su artículo 51, que la educación de los deficientes y de los inadaptados se llevará a cabo en centros ordinarios, en unidades de educación especial, y sólo en centros especiales cuando la profundidad de las anomalias que padezcan lo haga absolutamente necesario $^{2}$. Sin entrar a valorar la terminología de la época ni las estrategias organizativas que se proponen, en función de la gravedad de la deficiencia o inadaptación. La Ley, en ese el artículo, intenta suplir la desatención educativa de esos colectivos. Pese a todo, las circunstancias económicas y sociales del momento hicieron que la norma tardara en hacerse extensiva, cosa que también ocurrió con la educación de los superdotados, que hasta 1996 no sería legalmente asumida por el sistema educativo. No obstante representó, al igual que otros retos y mejoras educativas de la LGE, un avance en lo que debía ser la educación para todos. En cualquier caso aún no se avistaba a la educación inclusiva. 
Teresa Fontán Montesinos, Gisela Rivero Marrero

Romper el ciclo de la invisibilidad. El empleo como vía para favorecer la inclusión

A partir de 1975 los cambios sociales en el Estado Español (modificación del modelo de gobierno y de la estructura del Estado, especialmente), derivaron en una legislación educativa más acorde con los requerimientos del momento, y del futuro. Por el Decreto 1151/1975, de 23 de mayo, se crea el Instituto Nacional de Educación Especial (en adelante, INEE) dependiente del Ministerio de Educación y Ciencia que, a pesar de sus carencias económicas y estructurales, planificó la educación especial y ordenó la heterogeneidad de sus instituciones: fue clave en el desarrollo de la atención de los alumnos con discapacidad (Alonso y Araoz, 2011). Un año más tarde, con el Real Decreto 1023/ 1976, de 9 de abril, se funda el Real Patronato de Educación Especial al que se integran, además del INEE, los Ministerios de Educación y Ciencia, Hacienda, Gobernación, Justicia y Trabajo. De ese modo se consolida una infraestructura que coordinaba, por primera vez, ámbitos y servicios relacionados con la atención a las discapacidades. En 1978 el Real Patronato de Educación Especial se transforma en Real Patronato de Educación y Atención a Deficientes. Los Reales Decretos, 2276/1978 de 21 de septiembre y 2828/ 1978 de 1 de diciembre, lo configuran como un lugar para la colaboración de iniciativas públicas y privadas. La reina doña Sofía es la presidenta del Patronato, y las funciones de su Junta son, entre otras, las de estimular las medidas necesarias para la eficaz, prevención de deficiencias; asegurar la coordinación de todos los servicios que exigen la adecuada y múltiple atención a los deficientes; promover la calidady extensión de la educación especial, potenciando todas las actividades con ella relacionadas o complementarias; instar, por todos los cauces la rehabilitación global de los deficientes e impulsar la integración social de los deficientes, velando por la supresión de cuantas barreras dificulten dicha integración en cualquier ámbito (RD 2828/1978, art. 4).

Los esfuerzos y medidas anteriores superaron la desatención de los niños con discapacidad, y aunque los consideremos precedentes de la educación inclusiva, se desarrollaron desde el modelo médico, según el cual la discapacidad es un problema del niño, y hay que rehabilitarlo. Desde esa perspectiva, los niños especiales deben ir a escuelas especiales con profesores especialistas, separadas de las escuelas ordinarias para niños normales con profesorado ordinario. Se trata, de la derivación al sistema de escolarización del modelo médico o clínico en donde cualquier dificultad de aprendizaje es vista como un síntoma de un déficit, que debe ser diagnosticado y tratado. Afortunadamente ese modelo de segregación educativa irá evolucionando hacia la integración escolar, pero aún quedará mucho para el arraigo de la cultura de la igualdad y la inclusión educativa. Hasta mediados los años 80 la escolarización del alumnado con discapacidad, con programas especificos y diferentes a los del resto del alumnado, se realizaba de manera generalizada en centros segregados (Alonso y Araoz, 2011).

\subsection{Políticas de integración educativas: La Constitución y los años 80}

En los años 80, periodo conocido como transición a la democracia, el sistema educativo español se transformó de selectivo y de doble itinerario -instituciones, 
Teresa Fontán Montesinos, Gisela Rivero Marrero

Romper el ciclo de la invisibilidad. El empleo como vía para favorecer la inclusión

currículum y legislaciones diferentes para la Educación General y Especial- en otro integrador y comprehensivo. Lo hicieron posible la Ley 13/1982 de 7 de abril, de integración social de los minusválidos (en adelante, LISMI), y el Real Decreto 334/1985, de 6 de marzo, de Ordenación de la Educación Especial. Ambos mandatos legales generaron también, de algún modo, un cambio de actitudes en la atención social y educativa de las personas con problemas derivados de deficiencias físicas, psíquicas o sensoriales, y la adopción de planteamientos y soluciones más acordes con la dignidad, necesidades e intereses de las mismas.

En la Constitución Española de 1978 se expresa inequívocamente el derecho de todos a la educación (artículo 27), y se afirma que los poderes públicos están obligados a realizar una politica de previsión, tratamiento, rehabilitación e integración de los ciudadanos con minusvalias en todas las áreas sociales (artículo 49). El INEE tras la promulgación de la Constitución (1978), y en ese mismo año, elabora el Plan Nacional para la Educación Especial, en el que se formularon por primera vez los principios de: a) normalización de servicios, b) integración educativa, c) sectorización de la atención educativa, y d) individualización de la enseñanza. Dichos principios, entonces utópicos, alcanzaron el rango de norma, cuatro años más tarde, con la LISMI (1982).

La LISMI (1982) en su Título I, artículo 1, señala que los principios que la inspiran se fundamentan en los derechos que el artículo 49 de la Constitución reconoce, en razón a la dignidad que les es propia, a los disminuidos en sus capacidades físicas, psíquicas o sensoriales para su completa realización personal y su total integración social, y a los disminuidos profundos para la asistencia y tutela necesarias. Los beneficiarios de ese precepto constitucional lo son en razón de la existencia de una deficiencia que les impide o dificulta su inserción en la vida social. Es irrelevante, a los efectos de la protección de la norma, que la deficiencia sea de nacimiento o sobrevenida, así como que la causa de la dificultad de inserción provenga de un defecto físico o sensorial o de un problema psíquico, ya que lo que se valora es la existencia de la minusvalía permanente, de carácter congénito o no ${ }^{3}$. La LISMI (1982) establece el marco legal de esa protección y su valoración ${ }^{4}$. Fija en su Título VI, Sección $3^{\text {a }}$, artículos 23 al 31, los derechos de protección asistencial y de educación. La educación especial, congruente con esos derechos, tratará de conseguir la integración social del minusválido fundamentalmente a través de un empleo adecuado que les permita servirse y realizarse a sí mismos (Art.26) ${ }^{5}$

Los principios de la educación especial de la LISMI (1982) y del Plan Nacional para la Educación Especial (1978) son los mismos: a) normalización de los servicios, b) integración escolar, c) sectorización de la atención educativa, y d) individualización de la enseñanza. Una vez más se insiste que el alumno con minusvalías se integrará en el sistema ordinario de la educación general cuando reciba los apoyos necesarios que prevé la Ley. Además, conforme al principio de normalización, el alumno con minusvalía utilizará y recibirá servicios excepcionales sólo en los casos estrictamente imprescindibles. Consecuentemente se beneficiará, hasta donde sea posible, del sistema 
Teresa Fontán Montesinos, Gisela Rivero Marrero

Romper el ciclo de la invisibilidad. El empleo como vía para favorecer la inclusión

ordinario de prestaciones de la comunidad. En el ámbito educativo el principio de normalización se transforma en el de integración escolar; y en congruencia con el principio de sectorización, la atención educativa se acerca y se acomoda al entorno en donde el alumno con discapacidad desarrolla su vida; lo que supone ordenar los servicios educativos por sectores geográficos, de población y de necesidades. Finalmente, el principio de individualización de la enseñanza reclama que el centro de la atención educativa sea el sujeto, único e irrepetible, y que los procedimientos didácticos se adapten a su condición. Es necesario, pues, que cada alumno pueda encontrar la ayuda necesaria, la que necesita él justamente y no otra, para que el proceso de aprendizaje y desarrollo se realice de manera más eficaz (Castillejo, 1983, pág. 72). En resumen, aunque una ley no es un concepto, la indiscutible fuerza de los principios sobre los que se asienta: normalización de los servicios, integración escolar, sectorización de la atención educativa, e individualización han introducido una forma distinta de entender al alumno con minusvalías que se ha ido consolidando en un nuevo modelo de atención social y educativa.

En el mismo orden de ideas de rehabilitación de la LISMI (1982) ${ }^{6}$, el Real Decreto 334/1985, de 6 de marzo, de Ordenación de la Educación Especial, sienta las bases para conseguir la incorporación de los minusválidos a la vida social y a un sistema de trabajo que les permita servirse y realizarse a sí mismos. Prevé, en primer lugar, que la institución escolar ordinaria sea dotada de unos servicios que favorezcan el proceso educativo de todo el alumnado, eviten su segregación y faciliten su integración; en segundo lugar, que esa misma institución escolar contemple la existencia de centros específicos de educación especial, que aprovechen y potencien al máximo las capacidades de aprendizaje del alumno con minusvalías, y en tercer lugar, que entre los centros ordinarios y los de educación especial se establezca la coordinación necesaria. Con esas medidas se consideró que el primer paso para la integración del alumnado afectado por disminuciones físicas, psíquicas, sensoriales, o por inadaptaciones era su inserción completa o parcial en el sistema educativo ordinario, facilitada o posibilitada por los apoyos individualizados, prestados por personal especializado. Sólo, cuando las capacidades del sujeto NO permitieran dicha inserción, se recurriría a su escolarización en centros específicos. Una estrategia similar se adoptó con alumnos de otros grupos en situación de desigualdad, tal fue el caso de los de etnia gitana que se escolarizaban en concentraciones escolares separadas (siempre bajo la tutela del Ministerio de Educación), o los de clases sociales desfavorecidas que se orientaban hacia la enseñanza compensatoria o formación profesional. En cualquier caso las políticas de integración intentaba garantizar el derecho a la posterior escolarización en centros ordinarios: El derecho de todos los ciudadanos a la educación se hará efectivo, con respecto a las personas afectadas por disminuciones fisicas, psíquicas o sensoriales o por inadaptaciones, a través, cuando sea preciso, de la educación especial, como parte integrante del sistema educativo (LISMI, art.1). La respuesta a la diversidad en el aula, el cuestionamiento de la homogeneidad y la celebración de la diversidad, no serían motivo de preocupación hasta avanzados los 80, tras la puesta en marcha 
Teresa Fontán Montesinos, Gisela Rivero Marrero

Romper el ciclo de la invisibilidad. El empleo como via para favorecer la inclusión

en el 85 del Plan Nacional de integración escolar a escala nacional, y la aparición de los documentos e ideas que impulsó de manera muy clara el Centro Nacional de Recursos para la Educación Especial (CNREE) (Parrilla. 2007, pág. 3).

El desarrollo de la integración educativa recayó en los equipos multiprofesionales formados por profesionales de la medicina, psicología, pedagogía y trabajo social. Entre sus funciones se encontraban el promover y garantizar la incorporación de alumnos de centros de educación especial a los centros ordinarios. Un reto difícil. La integración educativa resultó episódica, de niño a niño y de profesor a profesor. En la práctica los apoyos legales fueron la autorización de la Administración, la colaboración de los Ayuntamientos, el seguimiento de la Inspección de Educación y el compromiso de las familias y del profesorado (general y especial). A veces lograr la coordinación de instituciones y personas tan diversas era difícil, y esa circunstancia, entre otras, hacía que la presencia de alumnos con discapacidad en las aulas ordinarias fuera bastante marginal, y cuando se conseguía la preocupación máxima era cómo incorporarlos a la cultura escolar. ${ }^{7}$

Consideramos que tanto la LISMI (1982) como el Real Decreto 334/1985 representan dos hitos en la educación española: la Ley por los principios que introdujo, y el Decreto porque estableció medidas para que los alumnos con discapacidad alcanzaran, en el máximo grado posible, los objetivos educativos establecidos con carácter general. Pero, a pesar de esas intenciones y normas, somos conscientes que poco se consiguió en la práctica. Tal vez la explicación de lo sucedido se encuentre, en primer lugar, porque las políticas de integración se sustentaron en principios -criterios siempre orientativos, moralmente importantes, que no comprometen a sus destinatarios-; en segundo lugar, porque el sistema educativo no se adaptó al niño con discapacidad sino que era él quien debía hacerlo, mediante la terapia o rehabilitación que unos especialistas dictaban para su curación, y si no la conseguía, fracasaba; y en tercer lugar, porque la organización y funcionamiento de los centros escolares ordinarios no se adaptaron a los alumnos con discapacidad, lo que propició que, salvo excepciones, esos alumnos fueran más agregados que integrados en sus aulas. Ahora bien, hay que reconocer que el desarrollo de esas políticas educativas hizo evidente que el proceso de integración escolar NO puede ser el fruto de la labor aislada de profesores sino de todo el centro escolar en colaboración con los equipos multiprofesionales y los servicios sociales. A pesar de todo, la superación del el modelo médico-rehabilitador de la discapacidad y la transformación de los centros escolares en comunidades educativas, adaptables y abiertas a la complejidad de una educación sin exclusiones, tardarían algo más en extenderse. 
Teresa Fontán Montesinos, Gisela Rivero Marrero

Romper el ciclo de la invisibilidad. El empleo como via para favorecer la inclusión

\subsection{Políticas de normalización e integración. Los años 90: inicio de la edu- cación inclusiva}

Los principios de normalización e integración de la LISMI (1982) y del Real Decreto 334/1985 dejaron de de ser un compromiso moral para los profesores cuando la Ley Orgánica 1/1990, de 3 de octubre, de Ordenación General del Sistema Educativo (en adelante, LOGSE) introdujo en el ordenamiento jurídico el concepto de necesidades educativas especiales (en adelante, NEE). El concepto de NEE tiene su origen en el Informe Warnock (1978) que propuso que la categorización tradicional de la discapacidad debía reemplazarse, y estableció que no era positivo continuar con la distinción entre alumnos con discapacidad y sin discapacidad, porque la educación especial debe entenderse como un continuo de prestación que va desde la ayuda temporal hasta la adaptación permanente o a largo plazo del currículum ordinario. Pero, a pesar de esa secuencia de prestación lineal, el término dificultad de aprendizaje se utilizará para describir la ayuda que algunos alumnos necesitan $^{8}$. El Informe Warnock (1978) amplió la educación especial no solo más allá de la referencia exclusiva a las personas con discapacidad sino que su concepto de NEE derivó a la adaptación y diversificación del curriculum ordinario: las prestaciones educativas especiales, donde quiera que se realicen tendrán un carácter adicional o suplementario y no alternativo o paralelo. De ese modo, la educación especial se va distanciando de planteamientos médicos o psicológicos de rehabilitación de déficit para ir situándose en los procesos educativos, y por tanto, en la adaptación del curriculum al alumno, y no del alumno al curriculum.

El concepto de NEE remite a una concepción interaccionista de la naturaleza de las diferencias individuales en lugar de la estática y de la ambientalista o situacional (Hunt y Sullivan, referenciado por Coll y Miras 2001) ${ }^{9}$. En la concepción interaccionista la persona con discapacidad y el entorno educativo son dos unidades separadas que interactúan, y esa acción reciproca supondrá relaciones causa-efecto entre las características de esas dos variables o dimensiones. Por tanto, a pesar de que esas características interactúan negativamente con algunos alumnos, generando en ocasiones barreras para su aprendizaje, no tienen por qué ser consecuencia exclusiva del individuo sino el resultado de esa interacción problemática. Por ello, en la acción educativa, las medidas para la eliminación de esas barreras deben dirigirse tanto a los aspectos propios del alumno como a los del contexto. El alumno con NEE no sólo es el que posee algún tipo de discapacidad, deficiencia o minusvalía, en terminología de la Organización Mundial de la Salud (1976), sino cualquiera que en su vida escolar se enfrente a barreras que le impidan el proceso de aprendizaje. El sistema educativo debe responder a la diversidad de alumnos pero cuestionando siempre cuales son las diferencias o características individuales que importan a la hora de enseñar y aprender; ¿las que configuran la inteligencia entendida en su dimensión simplemente cognitiva o académica o las que 
dan cuenta de las inteligencias múltiples que conforman la identidad de toda persona? (Gardner, 1995 y 2001).

En lo sucesivo todos los niños, en cualquier circunstancia, serán considerados educables, y la educación especial consistirá en la satisfacción de sus necesidades. A partir de ahora queda superada la concepción segregacionista de la educación especial por la educación como ayuda y orientación, intencional y sistematizada, que auxilia y potencia. Todos los alumnos necesitan esa ayuda, más o menos específica, para alcanzar los fines de la educación, pero ésta se realiza y es per se un proceso de perfeccionamiento individual ${ }^{10}$. Desde esos planteamientos la integración escolar se convierte en un modelo educativo que reclama la fusión de la educación general y la especial. Se defiende, por tanto, la unificación y reestructuración de ambas así como la participación de todos los alumnos en la propia comunidad (Stainback y Stainback, 1999). Ahora la preocupación es cómo avanzar hacia una educación para todos sin exclusiones. Pero esa aspiración está llena de dificultades, porque en último término, los modelos con los que analizamos y organizamos la realidad escolar tienen mucho que ver no sólo con la valoración de las diferencias humanas (género, identidad, capacidad, etnia,....) sino también de nuestra respuesta hacia ellas, como individuos y miembros de una institución escolar que desarrolla una determinada cultura (López Melero, 2001; Torres, 2002). Esa reacción emocional discurre o puede discurrir entre la segregación en categorías, la asimilación o integración al patrón dominante (lo masculino, lo occidental, lo sano, lo capaz, lo católico,...), o el respeto intercultural basado en el aprecio y la valoración de las diferencias como algo enriquecedor para todos (Gimeno, 2001). En definitiva son variables pedagógicas, organizativas y culturales las que configuran, en función de sus valores, los modelos educativos garantes del principio de igualdad en la diversidad.

Con la LOGSE (1990) la educación especial deja de concebirse como la educación para un tipo diferente de alumnos para entenderse como el conjunto de recursos materiales y personales puestos a disposición del sistema educativo para que éste pueda responder adecuadamente a las necesidades de todos los alumnos. Desde esta perspectiva, toda educación es siempre especial (educación para cada uno, individualizada, que ayuda y potencia en función de las necesidades), y es, el sistema educativo, el que debe disponer de los recursos necesarios para que todos los alumnos puedan alcanzarla, con ayuda y orientación, o sin ella. Ahora bien, la LOGSE (1990) establece, en su artículo 36, el marco adecuado para que el alumnado con necesidades educativas especiales, permanentes o transitorias, alcance los objetivos establecidos para todos los alumnos, y dispone que al final de cada curso escolar se evalúen los resultados tomando como referencia la valoración inicial, es decir, la mejora constatada en su proceso de aprendizaje.

La LOGSE (1990) considera que la educación escolar es el instrumento por excelencia para satisfacer las necesidades educativas de todos los alumnos sin excepción, 
Teresa Fontán Montesinos, Gisela Rivero Marrero

Romper el ciclo de la invisibilidad. El empleo como vía para favorecer la inclusión

compensar sus desigualdades y promover la integración y cohesión social. El centro escolar se convierte en una institución abierta a las diferencias en donde NO debe haber alumnos y alumnas "normales" y alumnas y alumnos "diversos" porque precisamente todos somos diferentes. Tener esto presente es importante porque choca con una concepción restringida de la diversidad muy extendida, según la cual, la "diversidad" se contrapone a la "normalidad" o sea se entiende que son "diversos" aquellos alumnos que no son "normales", cuando en realidad, la "diversidad" se identifica con la "normalidad, lo más normal es que somos diferentes" (Pujolás, 1998, p.15).

La LOGSE (1990) al optar por inclusión educativa introdujo en nuestro sistema educativo el éticamente controvertido dilema de las diferencias, que siempre necesitará un fortísimo apoyo social que contrarreste y ayude a cambiar las creencias y estereotipos negativos al respecto. El estudio de Sapon-Shevin (1996), que referencia Echeita (2010), señala algunos de los más habituales: que la inclusión educativa está siendo impuesta por ideologías extremas y por padres que no son realistas y no aceptan que sus hijos sean diferentes; que la inclusión educativa obligará a los profesores a rebajar el currículo, a que bajen los niveles educativos, limitando o perjudicando el aprendizaje de aquellos alumnos más capaces; que la inclusión educativa sólo tiene sentido para algunos alumnos pero no para otros difíciles de incluir o que no se debe hacer en todos la etapas educativas o que, finalmente, la inclusión educativa es un favor que se hace a determinados alumnos a costa de otros. Hoy por hoy sigue siendo difícil lograr el apoyo social mayoritario a la inclusión, y desde sus inicios se consideró que había que concederle no solo respaldo legislativo sino también evaluarla, y demostrar a la sociedad lo que se consigue con ella.

El Real Decreto 696/1995, de 28 de abril, de Ordenación de la educación de los alumnos con necesidades educativas especiales, con objeto de desarrollar los artículos 36 y 37 de la LOGSE, reguló la ordenación, la planificación de recursos y la organización de la atención educativa a los alumnos con necesidades educativas especiales, actualizando la normativa sobre integración escolar y educación especial. Dictaminó, en su artículo 7.2, la posibilidad de llevar a cabo adaptaciones curriculares significativas que afecten a los elementos prescriptivos del currículo, previa evaluación psicopedagógica realizada por los equipos de orientación educativa y psicopedagógica o, en su caso, por los Departamentos de orientación de los centros. Por su parte, los Reales Decretos $1333 /$ 1991, 1344/ 1991 y 1345/1991, de 6 de septiembre, que establecen, respectivamente, los currículos de Educación Infantil, Educación Primaria, y Educación Secundaria Obligatoria, consideran esa posibilidad de adaptación, y especifican que su ámbito se podría extender a la adecuación de los objetivos educativos, a la eliminación o inclusión de determinados contenidos y a la consiguiente modificación de los criterios de evaluación, así como a la ampliación de las actividades educativas de determinadas áreas curriculares. Asimismo, el Real Decreto 1179/1992, de 2 de octubre, por el que se establece el currículo del Bacbillerato, precisa que para el alumnado con problemas graves de audición, visión y motricidad, esas adaptaciones podrían extenderse a la exención total 
Teresa Fontán Montesinos, Gisela Rivero Marrero

Romper el ciclo de la invisibilidad. El empleo como vía para favorecer la inclusión

o parcial en determinadas materias, y el Real Decreto 676/1993, de 7 de mayo, por el que se establecen las directrices generales sobre los titulos y las correspondientes enseñanzas minimas de formación profesional, encomienda a las Administraciones educativas que fijen el marco que regule las posibles adaptaciones curriculares. Otra normativa, que también desarrolla los principios educativos de LOGSE, es la Orden de 14 de febrero de 1996 sobre evaluación de los alumnos con necesidades educativas especiales que cursan las enseñanzas de régimen general establecidas en la Ley Orgánica 1/1990, de 3 de octubre, por la que se regula el procedimiento de la evaluación psicopedagógica y el dictamen de escolarización, y se fijan los criterios para la escolarización de los alumnos con NEE.

La LOGSE (1990) certifica la desaparición de la educación especial como sistema educativo independiente y establece la fusión con la educación general en un único sistema, integrador y comprehensivo, que adopta un curriculum común. Sin embargo el foco del cambio educativo, centrado en el curriculum y en los alumnos individuales, fue un reto difícil para los profesores, porque a partir de ahora todos tenían que participar en el diseño y desarrollo de las adaptaciones del curriculum común a los contextos y necesidades del centro, del aula y de cada alumno en particular. Quizá por ese énfasis la cuestión de cómo elaborar Adaptaciones Curriculares Individuales para alumnos identificados con NEE se convierte en el punto nuclear de la respuesta a la diversidad (Parrilla, 2007, pág.6). Y pasó, a segundo plano, el cambio ideológico profesional que se necesita para articular un sistema de valores compartidos como respuesta institucional a la diversidad. Y en consecuencia, las Adaptaciones Curriculares Individuales, pensadas como respuesta equitativa a la diversidad, fueron a veces un impedimento a la misma al convertirse en un fin y no en un medio. $\mathrm{Y}$ así las cosas, los Proyecto Curriculares propios de cada centro, salvo experiencias aisladas, tuvieron un carácter más burocrático que real.

La educación inclusiva, pese a las dificultades de su desarrollo, supera las anteriores concepciones de integración educativa en tres puntos: a) Se dirige a toda la población excluida de la educación o con riesgo de serlo, b) Se focaliza en el centro escolar y, por extensión, en el sistema educativo, y c) Se concibe la inclusión como una tarea comunitaria. Afortunadamente, la educación inclusiva, a pesar de las luces y sombras de su puesta en práctica, ha conformado un marco más amplio para abordar la diversidad con expresiones como "atención educativa a la diversidad" y "respuesta a la diversidad" que hacen referencia a la nueva situación educativa caracterizada por la betereogeneidad y diferencias entre alumnos debidas no sólo a NEE sino a clase social, cultura o género (Parrilla, 2007, pág.6).

\subsection{Las dificultades y logros de la educación inclusiva: los inicios del siglo XXI}

La inclusión educativa de la LOGSE y de los decretos que la desarrollan se vieron amenazados por la Ley Orgánica 10/2002, de Calidad de la Educación (en adelante, 
Teresa Fontán Montesinos, Gisela Rivero Marrero

Romper el ciclo de la invisibilidad. El empleo como via para favorecer la inclusión

LOCE), que si bien no llegó aplicarse, en sus planteamientos se vislumbra un cierto retroceso con relación hasta lo entonces conseguido. La LOCE (2002) apuesta por la equidad que garantice una igualdad de oportunidades de calidad, para el pleno desarrollo de la personalidad a través de la educación, en el respeto a los principios democráticos y a los derechos y libertades fundamentales (art. 1a). La LOCE (2002) al apostar por la equidad, no considera la igualdad en la diversidad, pero sí admite que el alumnado con NEE tendrá una atención especializada, con arreglo a los principios de no discriminación y normalización educativa, y se le dotará, con la finalidad de conseguir su integración, del apoyo preciso desde el momento de su escolarización o de la detección de su necesidad (art. 44.1). En el Título II, Equidad en la Educación, se hace una distinción entre el alumnado con necesidad específica de apoyo educativo y la compensación de desigualdades en educación. Distingue tres grupos de alumnos: a) los de NEE (discapacitados sensoriales, psíquicos y físicos y con graves trastornos de la personalidad o de la conducta), b) los de altas capacidades intelectuales, y c) los de integración tardía en el sistema educativo español. Entre esos últimos, que son los que se atienden en compensatoria, se encuentran los inmigrantes, los procedentes de ambientes e riesgo de exclusión social o marginalidad, y las minorías culturales. Al igual que en la LOGSE (1990), la identificación y valoración de las necesidades educativas especiales la realizarán equipos de profesionales de distintas cualificaciones (art. 45. 2). Pero, sin embargo, en la LOCE (2002) aunque se alude a planes de actuación en relación con las necesidades educativas de cada alumno (art. 45.2) y las adaptaciones precisas (art. 45.3), no hay referencias explícitas a adaptaciones o diversificaciones curriculares como ocurría en la LOGSE (1990). En el caso que las adaptaciones curriculares sean necesarias se reservan exclusivamente para los alumnos con NEE y los de compensatoria. Además, en la LOCE (2002), la escolarización se estructura en las modalidades siguientes: a) grupos ordinarios, b) aulas especializadas en Centros ordinarios, c) Centros de educación especial, y d) escolarización combinada (art. 45.1). Mientras que en la LOGSE (1990) no se hacía alusión a la escolarización en aulas especiales en Centros ordinarios, ya que apostaba por una integración total en estos Centros. Por último, la LOCE (2002) dedica el (art. 48) a la integración social y laboral de este tipo de alumnado, defendiendo que con la finalidad de facilitar la integración social y laboral de los alumnos que no puedan conseguir los objetivos previstos en la enseñanza básica, las Administraciones públicas promoverán ofertas formativas adaptadas a las necesidades específicas de los alumnos.

En resumen, aunque la LOCE nunca llegó a aplicarse, se le reconoce como un exponente de la llamada modernización conservadora al estar forjada en torno a una amalgama de principios, no exentos de contradicciones internas, que tienen su origen en una visión de la vida social y de las prácticas educativas escolares de corte neoliberal, neoconservador, de populismo autoritario, de fanatismo religioso y gerencialismo (Apple, 2002). Pero, ¿no son esos los mismos principios de la Ley Orgánica 8/2013, de 9 de diciembre, para la mejora de la calidad educativa (LOMCE)?, ¿qué evidencia cuando modifica la Ley Orgánica 
Teresa Fontán Montesinos, Gisela Rivero Marrero

Romper el ciclo de la invisibilidad. El empleo como via para favorecer la inclusión

2/2006, de 3 de mayo, de Educación (LOE), y seis artículos y una disposición adicional de la Ley Orgánica 8/1985, de 3 de julio, reguladora del Derecho a la Educación (LODE)? Solo un matiz, la LOMCE no fue apoyada por ningún otro grupo parlamentario que el popular. Compartimos las críticas que se le hacen: La LOMCE no es más que la otra cara de los recortes educativos, que mira al pasado, que va a segregar alumnos desde muy jóvenes y además apoya a la escuela concertada y a la Iglesia ${ }^{11}$. Las amenazas de retroceso educativo y social por la LOMCE (2013) se confirman con el Real Decreto Legislativo 1/2013, de 29 de noviembre, por el que se aprueba el Texto Refundido de la Ley General de derechos de las personas con discapacidad y de su inclusión social (BOE de martes 3 de diciembre de 2013), cuando deroga y refunde gran parte de lo conseguido hasta ahora: a) La Ley 13/1982, de 7 de abril, de integración social de las personas con discapacidad (LISMI), b) La Ley 51/2003, de 2 de diciembre, de igualdad de oportunidades, no discriminación y accesibilidad universal de las personas con discapacidad (LIONDAU), y c) La Ley 49/2007, de 26 de diciembre, por la que se establece el régimen de infracciones y sanciones en materia de igualdad de oportunidades, no discriminación y accesibilidad universal de las personas con discapacidad.

Las actuales derogaciones y refundiciones legislativas nos hacen destacar aquello que la Ley 51/2003, de 2 de diciembre, de igualdad de oportunidades, no discriminación y accesibilidad universal de las personas con discapacidad (LIONDAU) completó y actualizó de la LISMI (1982). Introdujo, entre otras modificaciones, la denominación de personas con discapacidad en lugar de personas disminuidas físicas, psíquicas o sensoriales. Se inspiró en los principios de vida independiente, normalización, accesibilidad universal, diseño para todos, diálogo civil y transversalidad de las politicas en materia de discapacidad (Artículo 2) ${ }^{12}$. Concretó sus áreas de actuación en la igualdad de trato y no discriminación en materia de empleo y ocupación. Reguló el acceso al empleo como actividad profesional por cuenta propia y ejercicio profesional, la afiliación y participación en las organizaciones sindicales y empresariales, las condiciones de trabajo, la promoción profesional formación profesional ocupacional y continua. La LIONDAU (2003) también contó con una herramienta para realizar reclamaciones o quejas a la Administración, cuando fueran vulnerados los derechos como personas con discapacidad: La Ley 49/2007, de 26 de diciembre, por la que se establece el régimen de infracciones y sanciones en materia de igualdad de oportunidades, no discriminación y accesibilidad universal de las personas con discapacidad. Este sistema arbitral de quejas y reclamaciones en materia de igualdad de oportunidades, no discriminación y accesibilidad por razón de discapacidad garantizó los derechos en los sectores profesionales siguientes: telecomunicaciones y sociedad de la información, espacios públicos urbanizados, infraestructuras y edificación, transportes, bienes y servicios a disposición del público, relaciones con las Administraciones Públicas. A partir de ahora, no sabemos hasta qué punto con el nuevo el ordenamiento jurídico español, las personas con discapacidad tendrán garantizadas la igualdad de oportunidades, la no discriminación y la accesibilidad universal, y las posibilidades de reclamación de sus derechos. 


\section{Teresa Fontán Montesinos, Gisela Rivero Marrero}

Romper el ciclo de la invisibilidad. El empleo como via para favorecer la inclusión

Entre las dificultades y logros de la educación inclusiva, en los inicios del siglo XXI, no queremos dejar de mencionar la Ley Orgánica 2/2006, de 3 de mayo, de Educación (en adelante, LOE), que contribuyó a facilitar la generalización de la educación inclusiva (Casanova, 2011) al proporcionar a los centros educativos no sólo la autonomía sino también la flexibilidad en la aplicación de la norma al entorno y a las características de la población que atiende. En la LOE (2006) se consideró la Educación especial como parte de las enseñanzas de régimen general, que se inspiraba, según sus artículos 1 y 2, en los principios y fines siguientes:

Son principios del sistema educativo:

- La calidad de la educación para todo su alumnado, con independencia de sus condiciones y circunstancias.

- La equidad, que garantice la igualdad de oportunidades, la no discriminación e inclusión educativa como elementos compensadores de desigualdades personales, con atención especial a las que se derivan de la discapacidad.

- La flexibilidad para adaptar la educación a la diversidad de aptitudes, intereses, expectativas y necesidades del alumnado.

Son fines del sistema educativo:

- El desarrollo pleno de la personalidad y capacidad del alumnado.

- La educación en la igualdad de trato y no discriminación de las personas con discapacidad.

La LOE (2006) dedicó el capítulo I, de su Título II a la atención del alumnado con necesidad específica de apoyo educativo, de origen diverso: incorporación tardía al sistema educativo, altas capacidades, o condiciones personales o de historia escolar. Cuando derivaban de discapacidades o trastornos graves de conducta las denominaba necesidades educativas especiales (art.73). Y en cualquier circunstancia todas las enseñanzas se debían adaptar al alumnado con necesidad especifica de apoyo educativo para asegurar su acceso, permanencia y progresión en el sistema educativo (art. 3.8). Y de acuerdo con el (art. 7.), la escolarización del alumnado de necesidades educativas especiales, tendría las características siguientes: a) se regirá por los principios de normalización e inclusión educativa, b) asegurará su no discriminación y la igualdad en el acceso y la permanencia en el sistema educativo, introduciendo las medidas necesarias en las distintas etapas educativas, y c) sólo se llevará a cabo en unidades o centros de educación especial, cuando sus necesidades no puedan ser atendidas por las medidas de atención a la diversidad de los centros ordinarios.

En los años de aplicación de la LOE los profesionales de la educación han sido cada vez más conscientes que la atención a la diversidad exige una mayor competencia 
Teresa Fontán Montesinos, Gisela Rivero Marrero

Romper el ciclo de la invisibilidad. El empleo como via para favorecer la inclusión

profesional, proyectos educativos más complejos, capacidad de adaptar el currículo a las necesidades educativas de los alumnos y una mayor provisión de recursos de todo tipo. Ha quedado claro que para dar respuesta a la compleja situación de la educación de alumnos con NEE en contextos ordinarios, hay que trabajar en las instituciones educativas no sólo colaborativamente sino también traspasando las fronteras disciplinares y profesionales. Eso sí, sin olvidar nunca el sentido educativo de la investigación para la mejora de la práctica. Se trata de reconocer la diversidad como un valor y no como un defecto. Implica romper con la clasificación y la norma, supone plantearnos una necesaria profesionalización del docente para la comprensión de la diversidad y requiere pensar en un currículo que, ahondando en las diferencias del alumnado, erradique las desigualdades a la vez que haga avanzar la justicia escolar ofreciendo prácticas educativas simultáneas y diversas (López Melero, 2001). En síntesis, la educación inclusiva permite no solo la preparación para que la persona con diversidad funcional se integre a la sociedad y disfrute de los avances científicos-tecnológicos sino que también la sociedad la reciba y esté dispuesta a brindarle cuantos recursos necesite ${ }^{13}$. ¿Qué ocurrirá a partir de ahora? El futuro está lleno de incertidumbre, pero afortunadamente la obligación y promoción de la educación inclusiva no radica exclusivamente en la legislación española sino también en normativas internacionales (declaraciones, convenciones, etc.), que esperamos se respeten y acaten.

\subsection{La inclusión educativa en las normativas internacionales}

Citamos por relevante la Convención de las Naciones Unidas sobre los derechos de las personas con discapacidad de 2006, firmada por la Unión Europea, y que obliga a todos los Estados miembros. La Convención dedica su artículo 24 a la educación, y aborda la inclusión de las personas con discapacidad en cualquiera de los aspectos de la vida: trabajo, vivienda, justicia, ocio, salud, etc. Y puntualiza que el derecho a la educación de las personas con discapacidad tiene tres objetivos: a) Desarrollar plenamente el potencial bumano y el sentido de la dignidad y de la autoestima y reforzar el respeto por los derechos humanos, las libertades fundamentales y la diversidad humana; b) Desarrollar al máximo la personalidad, los talentos y la creatividad de las personas con discapacidad, asi como sus aptitudes mentales y físicas; y c) Hacer posible que las personas con discapacidad participen de manera efectiva en una sociedad libre (UNITED NATIONS 2006, p.18).

Aunque la Convención entró en vigor el 3 de mayo de 2008, hay voces, como las de algunos colectivos de personas con discapacidad, que consideran que desde hace ya bastantes años la Convención (2008) está estancada (si no en retroceso). La educación inclusiva en España sigue siendo más una declaración de intenciones que un logro del que sentirse razonablemente satisfechos, sin perder de vista que se trata, en todo caso, de un viaje interminable (Echeita, 2010). Compartimos no solo la anterior valoración 
Teresa Fontán Montesinos, Gisela Rivero Marrero

Romper el ciclo de la invisibilidad. El empleo como via para favorecer la inclusión

sino también sus criterios, cuando señala que la causa de tal situación no es sólo estructural sino también porque falta convicción, determinación y voluntad política para acometer y sostener los cambios educativos que se precisan para ser coherentes con el hecho de haber sido, no sólo uno de los países impulsores de la Convención, sino de los primeros en firmarla y ratificarla. En efecto, este loable compromiso demandaba, al menos, NO seguir manteniendo en nuestro sistema educativo el sistema dual de escolarización entre centros ordinarios y centros específicos, lo cual contraviene el artículo 24 de la Convención, dedicado al derecho a la educación. Denunciamos que aún hoy la normativa de escolarización, en la mayoría de las Comunidades Autónomas españolas, se sigue basando sobre el criterio -propio del modelo médico de la discapacidad-, de que hay algunos alumnos que, por sus necesidades educativas no se pueden integrar, y por ello precisan, necesariamente, ser escolarizados en centros específicos. Cosa que muchas veces ocurre sin que la evaluación psicopedagógica de los servicios de orientación educativa haya facilitado u orientado los ajustes razonables en los centros ordinarios y, solamente se limiten a constatar aquellos déficits y dificultades del alumnado que hacen imposible su inclusión. Pero, si mal está que dicha evaluación no cumpla las expectativas para las cuales fue establecida normativamente, lo que resulta hiriente es que los equipos docentes de tantos y tantos centros escolares (más entre los centros concertados que entre los centros públicos), hayan reducido su obligación de realizar tales ajustes razonables. El círculo vicioso está cerrado: si no se hacen ajustes razonables, siempre habrá razones para que la inclusión educativa no sea posible para algunos estudiantes. Sin duda, esta ausencia de ajustes razonables es una pieza angular para entender la situación actual de la educación inclusiva, pero también para focalizar sobre ella nuestras expectativas de mejora, empezando por reclamar la tutela judicial frente a la discriminación, directa e indirecta, que produce su incumplimiento. En efecto, la perspectiva o el modelo de derechos, en los que está basada la Convención, obliga es a poner el foco de acción en la tarea de romper las barreras, de distinto tipo y condición, que impiden el ejercicio efectivo y pleno de los derechos reconocidos. Lo que falta es una instancia que obligue a que se cumpla la ley. Estimamos que tanto los servicios de inspección educativa como los tribunales de justicia tienen esa responsabilidad, pero no exclusiva. A medio y corto plazo también la tienen los formadores de los futuros profesores, las familias y las organizaciones de personas con diversidad funcional ${ }^{14}$. La educación inclusiva es el derecho de todos, sin exclusiones, a acceder, permanecer y progresar satisfactoriamente en un centro ordinario, donde aprender las competencias necesarias para la vida, sobre la base del reconocimiento y la valoración de la diversidad humana. ¿Se agravará la situación que denunciamos con la implantación de la LOMCE? Los recortes económicos ya se han hecho sentir. 
Teresa Fontán Montesinos, Gisela Rivero Marrero

Romper el ciclo de la invisibilidad. El empleo como vía para favorecer la inclusión

\section{El FUtURo INMEDiato de LA EDUCACión INCLUSIVA EN EsPaÑa}

El viaje interminable de la educación inclusiva en España, con sus luces y sombras, se ve peligrar con la derogación de lo conseguido hasta ahora. La lectura de la LOMCE (2013), desde la primera frase del preámbulo, nos llena de inquietud cuando se afirma que la educación es el motor que promueve la competitividad de la economía y el nivel de prosperidad de un pais. Es cierto, una mayor y mejor inversión en educación, refleja una preocupación por esta materia y por lograr que todo el alumnado tenga la mayor y mejor cualificación posible. Por ende, esa cualificación debería revertir en el progreso económico del país. Sin embargo, sólo cuatro párrafos más abajo, se puntualiza: La calidad educativa debe medirse en función del output (resultados de los estudiantes) y no del input (niveles de inversión, número de profesores, número de centros, etc.). Aquí es cuando comenzamos a no comprender el planteamiento inicial. Se reconoce que la educación es el motor de la competitividad de la economía y de la prosperidad del país, pero se recortan millones de euros en inversión para educación e investigación, hay menos profesorado, se cierren centros públicos en beneficio de entidades privadas, etc. ${ }^{15}$ Pese a todo, se insiste en el preámbulo de la LOMCE que lo más importante son los resultados del alumnado: Todos los estudiantes poseen talento, pero la naturaleza de este talento difiere entre ellos, por lo que el sistema educativo debe contar con los mecanismos necesarios para reconocerlos y potenciarlos. Más adelante se habla de un sistema capaz de canalizar a los estudiantes hacia trayectorias más adecuadas a sus fortalezas. Pero, ¿cuál es esta canalización?: a) Una formación profesional básica que sustituye a los Programas de Cualificación Profesional Inicial (PCPI) y que no conduce a la titulación de Graduado en ESO; por lo tanto, el alumnado que con 14 o 15 años se equivoque, no se esfuerce lo suficiente por los avatares de la adolescencia o, simplemente, atraviese circunstancias que le impidan dedicarse más intensamente a los estudios, podría ver en esta vía el final sus posibilidades de desarrollo académico, y b) Un ciclo de educación secundaria obligatoria (de tres cursos) y un cuarto curso que se estudiará en la opción de enseñanzas académicas o enseñanzas aplicadas. La primera de las alternativas, conducirá a los estudios universitarios (previa superación del Bachillerato) y la segunda a la actividad profesional. Por tanto, con esta "clasificación" de alumnos basada en los "resultados de aprendizaje" peligra, entre otros, el cumplimiento y los valores educativos del artículo tercero del Marco de Acción de la Conferencia de Salamanca que dice: (...) las escuelas tienen que encontrar la manera de educar satisfactoriamente a todos los niños y niñas, incluidos aquellos con discapacidades graves,(...) El mérito de estas escuelas no es sólo que sean capaces de dar una educación de calidad a todos los niños; con su creación se da un paso muy importante para intentar cambiar las actitudes de discriminación, crear comunidades que acojan a todos y sociedades integradoras (UNESCO, 1994, pp. 59-60). De la reforma actual de nuestro sistema educativo extraemos una conclusión: NO es la vía hacia una educación inclusiva. Se trunca o se hace más difícil el camino hacia una sociedad más 
Teresa Fontán Montesinos, Gisela Rivero Marrero

Romper el ciclo de la invisibilidad. El empleo como vía para favorecer la inclusión

justa e integradora. Pero la última y quizás más desconcertante paradoja es que peligre lo conseguido hasta ahora cuando se conocen suficientemente las principales condiciones y factores que contribuyen al desarrollo de políticas y prácticas inclusivas (Ainscow y Milles, 2009).

3. UNOS DATOS SOBRE EL ESTADO DE LA EDUCACIÓN INCLUSIVA EN ESPAÑA

\begin{tabular}{lccccccr}
\hline & Total & E. Infantil & E.Primaria & ESO & PCPI & Bachillerato & F.P. \\
\hline Total & 1,9 & 0,8 & 2,0 & 2.0 & 6,4 & 0,2 & 0,3 \\
\hline Centros Públicos & 2,0 & 1,0 & 2,4 & 2,1 & 5,2 & 0,2 & 0,3 \\
$\begin{array}{l}\text { Enseñanza concertada } \\
\text { Enseñanza privada }\end{array}$ & 2,0 & 0,6 & 1,4 & 1,8 & 10,1 & 0,2 & 0,3 \\
$\begin{array}{l}\text { no concertada } \\
\text { Hombres }\end{array}$ & 0,2 & 0,1 & 0,2 & 0,2 & 2,4 & 0,1 & 0,1 \\
Mujeres & 2,3 & 1,0 & 2,5 & 2,4 & 5, & 0,2 & 0,3 \\
\hline
\end{tabular}

Fuente: Ministerio de Educación (2011): Datos y cifras de la educación en España. Curso escolar 2011-12. (pág. 6)

La tabla anterior recoge las cifras oficiales, del Ministerio de Educación, del porcentaje de alumnos con necesidades educativas especiales, matriculados en el Curso 2009-2010, incluyendo en el cálculo del Total el de Educación Especial específica. Su análisis nos suministra la información siguiente:

a) Que los alumnos con necesidades educativas especiales, incluidos los de Educación Especial específica, representan de 1,9\% de la población matriculada. Deducimos por ese cómputo global diferenciado que se mantiene un sistema de clasificación: a) necesidades educativas especiales y b) educación especial específica.

b) Se nos informa sobre la titularidad de los centros en los que se matriculan: públicos, concertados o privados, pero no se especifica en qué modalidad de escolarización: centros ordinarios, centros específicos, aulas específicas o enclaves en centros ordinarios. Es decir, si se trata de una escolaridad inclusiva o segregada.

c) Los datos globales disponibles muestran que el proceso de acceso, permanencia y progresión de alumnos con necesidades educativas especiales debe mejorar. El porcentaje menor de alumnado escolarizado se encuentra en Educación Infantil $(0,8 \%)$, y es inferior -en 1,2 punto porcentual-a los de las etapas siguientes de escolaridad obligatoria (Educación Primaria y Educación Secundaria Obligatoria: ESO), que tienen una ratio de matriculación equivalente $(2,0 \%)$. 
Teresa Fontán Montesinos, Gisela Rivero Marrero

Romper el ciclo de la invisibilidad. El empleo como vía para favorecer la inclusión

d) La tasa más elevada de alumnado con necesidades educativas especiales corresponde a los Programas de Cualificación Profesional Inicial -PCPI- $(6,4 \%)^{16}$. Los que consiguen cursar Bachillerato $(0,2 \%)$ lo hacen en un porcentaje menor que los de Formación Profesional-FP- (0,3\%). Pero al presentar los datos globales de matriculación no podemos inferir qué tipo de escolarización están recibiendo los alumnos, aunque es muy significativa la diferencia que existe entre los Programas de Cualificación Profesional Inicial -PCPI- (6,4\%) y la Formación Profesional $(0,3 \%)$.

Los datos muestran una valoración general insatisfactoria de la educación inclusiva en España, pese a las declaraciones y los instrumentos jurídicos que la respaldan. Persisten barreras para el acceso a la Educación Infantil y, sobre todo, para la progresión hacia las etapas de la educación secundaria postobligatoria (Bachillerato y Formación Profesional). El número de alumnos permanece constante en la educación obligatoria (Primaria y Secundaria) incrementándose significativamente en los Programas de Cualificación Profesional Inicial (PCPI). Consideramos que esta situación puede repercutir de manera decisiva en el desarrollo personal y la capacitación del alumnado, condicionando sus oportunidades futuras de formación y de acceso al entorno laboral, y por lo tanto a una vida lo más independiente y normalizada posible.

El porcentaje de alumnado con necesidades educativas especiales, en el curso 2009-10, nos hacen reflexionar sobre las distancias y encuentros entre la perspectiva conceptual de la educación inclusiva que defendemos y la estadística oficial disponible. El alumnado con necesidades educativas especiales sufre barreras escolares tanto por el sistema dual de clasificación como por las circunstancias de su acceso, permanencia y progresión en las etapas iniciales del sistema educativo. Lo interesante aquí sería que este esbozo situacional abriera al debate acerca de qué educación especial pensamos y qué educación especial se desprende de los datos disponibles. A continuación proponemos algunos ejes para seguir pensando:

El sujeto de la educación especial es el alumno con NEE. Este concepto no deja de lado la discapacidad, sino que la considera junto con los demás factores que inciden en la vida del individuo: ¿por qué no disponemos de información desde esta concepción? ¿A qué se debe que se incluya en los datos globales de alumnos con necesidades educativas especiales, los alumnos de Educación Especial específica? ¿Por qué no se diferencian estadísticamente? No sabemos a quién agrupa, qué tipo de problemática o tipo de discapacidad revela esta información estadística global del Ministerio de Educación.

Cabe preguntarnos: ¿si la información tiene como una de sus funciones el conocimiento y la toma de decisiones, qué podríamos esperar de la información? ¿qué deberíamos preguntar para "ir más allá" de la discapacidad? ¿son útiles estos datos para la gestión de la educación inclusiva?

El Guiniguada, No 22 (2013) • Las Palmas de Gran Canaria 


\section{Teresa Fontán Montesinos, Gisela Rivero Marrero}

Romper el ciclo de la invisibilidad. El empleo como vía para favorecer la inclusión

Sin quitar un ápice de importancia a la necesidad de seguir investigando y aprendiendo más sobre el proceso de la inclusión educativa, NO dejamos de lado la importancia de su estadística para representar su realidad y transformarla, predecir su futuro o simplemente conocerla. Pero es imprescindible que esa búsqueda de repuesta a muchas de las necesidades que nos plantea la educación inclusiva se dirija también a conseguir una profunda y extensa alfabetización ética de la sociedad y del mundo educativo en particular. Los valores son la base de todas las acciones y planes de acción, de todas las prácticas en los centros docentes y de todas las políticas que modelan las prácticas. No podemos adoptar un comportamiento correcto en la educación sin comprender, en cierto modo, los valores de los que nacen nuestras acciones. Por lo tanto, el desarrollo de la inclusión educativa nos implica a nosotros mismos a la hora de hacer explicitos los valores que subyacen a ella de la mejor manera posible (Booth, T. 2006). La educación inclusiva es la única educación moralmente deseable (Escudero y Martínez, 2011), y su sentido debe estar en el corazón del sistema educativo y presente en todos sus elementos (currículo, organización escolar, financiación, supervisión, etc.). Pero, en último término, son nuestros valores y principios (igualdad en las diferencias, equidad, no discriminación, responsabilidad, justicia...) el motor de nuestra lucha por una educación más inclusiva, y del empleo como vía para favorecer la inclusión de los alumnos en riesgo de exclusión social.

\section{BibLIOGRAFÍA}

AINSCOW, M. (1995). Necesidades especiales en el aula. Guia para la formación del profesorado. Madrid: Narcea.

AINSCOW, M., y Miles, S. (2009). Desarrollando sistemas de educación inclusiva. ¿Cómo podemos hacer progresar las políticas? En C. Giné, (coord). La educación inclusiva. De la exclusión a la plena participación de todo el alumnado (pp. 161-170). Barcelona: Horsori.

BOOTH, T. (2006). Manteniendo el futuro con vida; convirtiendo los valores de la inclusión en acciones. En M. A. Verdugo y F.B. Jordán (coords.)Rompiendo inercias. Claves para avanzar. VI Jornadas Cientificas de Investigación sobre Personas con Discapacidad (pp.211-217). Salamanca: Amarú.

CASTILLEJO, J. L. (1983). Nuevas perspectivas en Ciencias de la Educación. Madrid: Anaya.

COLL, C. y MIRAS, M. (2001). Diferencias individuales y atención a la diversidad en el aprendizaje escolar. En C. Coll, J. Palacios y A. Marchesi, (Comp). Desarrollo psicológico y educación, Vo.2 (pp. 331 356). Madrid: Alianza.

ECHEITA, G.; SIMÓN, C. y otros (2009). Paradojas y dilemas en el proceso de inclusión educativa en España. Revista de Educación, 349, 153-178.

ECHEITA, G. (2010). Alejandra L. S. o el dilema de la inclusión educativa en España. En P. Arnaiz, Mª D. Hurtado, y F. J. Soto (Coords.). 25 Años de Integración Escolar en España: Tecnología e Inclusión en el ámbito educativo, laboral y comunitario. Murcia: Consejería de Educación, Formación y Empleo.

ESCUDERO, J, M. y MARTÍNEZ, B. (2011). Educación inclusiva y cambio escolar. Revista Iberoamericana de Educación, 55, 85-105.

FERNÁNDEZ ENGUITA, M. (1999). La escuela como organización: agregado, estructura y sistema. Revista de Educación, 320, 255-267.

GARDNER, H. (1995). Inteligencias múltiples. La teoría en la práctica. Barcelona: Paidós. 


\section{Teresa Fontán Montesinos, Gisela Rivero Marrero}

Romper el ciclo de la invisibilidad. El empleo como vía para favorecer la inclusión

GARDNER, H. (2001). La inteligencia reformulada. Las inteligencias múltiples en el siglo XXI. Barcelona: Paidós.

GIMENO, J. (2001). Políticas de la diversidad para una educación democrática igualitaria. En A. Sipán (Coord.) Educar para la diversidad en el siglo XXI. Zaragoza: Mira Editores.

LÓPEZ MELERO, M. (2001). La cultura de la diversidad o el elogio de la diferencia y la lucha por las desigualdades. En A. Sipán (Coord.) Educar para la diversidad en el siglo XXI. Zaragoza: Mira Editores.

TORRES, J. (2002). La cultura escolar. Cuadernos de Pedagogía, 311, 71-75.

PUJOLAS, P. (1998). La atención a la diversidad. Dossier de la Maestría en Educación Especial e Integración Escolar. Universidad de Girona. España.

RUIZ, R., y GINÉ, C. (1986) Las necesidades educativas especiales. Cuadernos de Pedagogía, 139, 32-34.

STAINBACK, S. y STAINBACK, W. (Eds.) (1999). Aulas inclusivas. Madrid: Narcea.

SANDOVAL, M.; LÓPEZ, M. L. y otros (2002). Index for inclusion. Una guía para la evaluación y mejora de la educación inclusiva. Contextos educativos, 5, 227-238.

WARNOCK, M. H. (1978). Special Educational Needs: Repport of the Committee of Enquiry into the Education of Handicapped Children and Young People. London: HMSO.

\section{Fuentes electrónicas}

ALONSO, Má. J. y ARAOZ, I. (2011). El impacto de la convención Internacional de la Personas con Discapacidad en la legislación educativa española. Madrid, Ediciones Cinca. http:/ /www.convenciondiscapacidad.es/ Colección ON.

BOOTH, T. y AINSCOW, M. (2002). Index for Inclusion. Traducción castellana Guía para la evaluación y mejora de la educación inclusiva. Madrid: Consorcio Universitario para la Educación Inclusiva. www.uam. es/personal_pdi/stmaria/sarrio/DOCUMENTOS,\%2520ARTICULOS

EUROPEAN AGENCY FOR DEVELOPMENT IN SPECIAL NEEDS EDUCATION (2006). Educación Especial en Europa (Volumen 2) Factores clave en la educación post-primaria. www.european-agency.org/

PARRILLA, A. (2007). Inclusive Education in Spain: a view from inside. En L. Barton, y L. Armstrong (Eds). Policy, Experience and Change: Cross Cultural Reflections on Inclusive Education. Dordrecht, Springer Ed., pp 19-36. bttp://prometeo.us.es/idea/publicaciones/angeles/2.pdf

PUIGDELLÍVOL, I. (2009). Escuela inclusiva: ¿necesidades educativas especiales? g02.berritzeguneak.net/

TOBOSO, M., y FERREIRA, M., y otros (2012). Sobre la educación inclusiva en España: políticas y prácticas. Intersticios: Revista Sociologica de Pensamiento Crítico, vol. 6 no 1 - 2012 - 17pp. bttp:/ / digital.csic.es/ bitstream/10261/45303/1/Toboso-et-al._Intersticios_6-1.pdf

SANDOVAL M., ECHEITA G., SIMÓN C., y LÓPEZ M. Educación Inclusiva: iguales en la diversidad. Módulos de formación (1-9). http:/ / mmm.ite.educacion.es/formacion/materiales/126/cd/ unidad_1.

UNESCO (1994). Informe Final. Conferencia mundial sobre las necesidades educativas especiales: acceso y calidad. UNESCO/Ministerio de Educación y Ciencia, Madrid. bttp:// unesdoc.unesco.org/images/0011/ 001107/ 110753so.pdf

UNITED NATIONS (2006). Convención de las Naciones Unidas sobre los derechos de las personas con discapacidad. http:// wmw.un.org/spanish/disabilities/ 


\section{Teresa Fontán Montesinos, Gisela Rivero Marrero}

Romper el ciclo de la invisibilidad. El empleo como vía para favorecer la inclusión

\section{NOTAS}

1 Entendemos el empowerment como el proceso educativo que conduce a la realización personal e integración social de todos los niños, incluidos aquellos con discapacidades graves, porque ese es, entre otras razones, el camino para una sociedad más justa e integradora.

2 Ley General de Educación, Articulo 51: la educación de los deficientes e inadaptados, cuando la profundidad de las anomalias que padezcan lo haga absolutamente necesario se llevará a cabo en centros especiales, fomentándose el establecimiento de unidades de educación especial en Centros docentes de régimen ordinario para los deficientes leves cuando sea posible.

3 La Organización Mundial de la Salud (OMS), en su Clasificación Internacional de 1976, diferencia los supuestos de deficiencias, discapacidades y minusvalías. Define la minusvalía como la situación desventajosa para un individuo determinado, consecuencia de una deficiencia o de una discapacidad que limita o impide el desempeño de un papel que es normal en su caso, en función de la edad, sexo y factores sociales y culturales. La definición anterior será tenida en cuenta para reconocer a los titulares los derechos de protección, tanto en la Constitución (art. 49) como en la LISMI (art. 7.1): A los efectos de la presente Ley se entenderá por minusválidos toda persona cuyas posibilidades de integración educativa, laboral o social se ballen disminuidos como consecuencia de una deficiencia, previsiblemente permanente, de carácter congénito o no, en sus capacidades fïsicas, psíquicas o sensoriales.

4 En la LISMI (1982) la protección se cataloga como: a) protección directa, en forma de prestaciones sociales y económicas (asistencia sanitaria y farmacéutica, rehabilitación médico-funcional, recuperación profesional, subsidios en concepto de ingresos mínimos, de ayuda de terceras personas, de movilidad, servicios de atención integral domiciliaria, etc.); b) protección por medio de la integración laboral (ayudas de empleo a trabajadores minusválidos, reservas de puestos de trabajo en empresas, creación de centros especiales de empleo); y c) protección genérica (supresión de barreras arquitectónicas, vivienda, residencias especializadas).

5 LISMI, Articulo 26. Uno. La educación especial es un proceso integral, flexible y dinámico, que se concibe para su aplicación personalizada y comprende los diferentes niveles y grados del sistema de enseñanż, particularmente los considerados obligatorios y gratuitos, encaminados a conseguir la total integración social del minusválido. Dos. Concretamente, la educación especial tenderá a la consecución de los siguientes objetivos: a) La superación de las deficiencias y de las consecuencias o secuelas derivadas de aquéllas, b) La adquisición de conocimientos y hábitos que le doten de la mayor autonomía posible, c) La promoción de todas las capacidades del minusválido para el desarrollo armónico de su personalidad, y d) La incorporación a la vida social y a un sistema de trabajo que permita a los minusválidos servirse y realizarse a sí mismos.

6 El concepto de rehabilitación de la LISMI (1982) se recoge su Artículo 18. Uno. Se entiende por rehabilitación el proceso dirigido a que los minusválidos adquieran su máximo nivel de desarrollo personal y su integración en la vida social, fundamentalmente a través de la obtención de un empleo adecuado. Dos. Los procesos de rehabilitación podrán comprender: a) Rehabilitación médico-funcional, b) Tratamiento y orientación psicológica, c) Educación general y especial, y d) Recuperación profesional. Tres. El Estado fomentará y establecerá el sistema de rehabilitación, que estará coordinado con los restantes servicios sociales, escolares y laborales, en las menores unidades posibles, para acercar el servicio a los usuarios y administrado descentralizadamente.

7 La cultura escolar se define como los patrones de significado que son transmitidos históricamente, y que incluyen las normas, los valores, las creencias, las ceremonias, los rituales, las tradiciones, y los mitos comprendidos, quizás en distinto grado, por los miembros de la comunidad escolar (Stolp y Smith 1994).

8 El Informe Warnock (1978) analiza la situación de la Educación Especial en Inglaterra, y considera que uno de cada cinco niños (20\%) necesita, en algún momento de su vida escolar, una ayuda educativa especial. Sus recomendaciones han servido no sólo de referencia legislativa a distintos países,

El Guiniguada, No $22(2013) \cdot$ Las Palmas de Gran Canaria 


\section{Teresa Fontán Montesinos, Gisela Rivero Marrero}

Romper el ciclo de la invisibilidad. El empleo como vía para favorecer la inclusión

entre ellos España, sino que también han orientado a números especialistas: Hegarty (1981), Hodgson (1984), Brennan (1985), etc.

9 Para la concepción interaccionista las características individuales y las de la situación interactúan. La cantidad y la calidad de los aprendizajes no pueden ser atribuidos únicamente al alumno -nivel intelectual, conocimientos previos, motivación, intereses por el contenido, autoconcepto, etc.-; ni tampoco a las situaciones educativas en las que participa. Es en la interacción entre unas y otras, en el mayor o menor grado de ajuste entre ambas, donde hay que buscar la explicación de la cantidad y calidad de los aprendizajes.

10 Etimologicamente el término educación deriva de dos palabras latinas que le dotan de dos significados: Educare. Significa conducir a partir de. Es una acción externa, de influencia de uno sobre el otro, que bace referencia a proceso y a cambio. Educere. Significa hacer salir. En este aspecto se insiste en el proceso, pero no niega la influencia externa, aún cuando se admite la posibilidad de que la acción puede ser uno mismo. Es más extraer algo del hombre. Ambas significaciones nos enfrenta a una doble vía de perfección. Por una parte, el propio hombre se perfecciona en tanto en cuanto es capaz. de desenvolverse o de desarrollar sus propias posibilidades, de tal suerte, que en si mismo está la posibilidad de perfección. Por otra parte, la acción externa de conducir a otro, hacia niveles superiores de perfección es la vertiente de ayuda y orientación de la educación (Castillejo, J.L. 1983: 17). No nos adscribimos sectariamente a una de las posturas, consideramos que la influencia externa, intencional y sistematizada, auxilia y potencia la educación, pero ésta se realiza y es per se un proceso de perfeccionamiento individual de todos los alumnos.

11 La Iglesia gana la reforma educativa. Periódico el País, de 17 de mayo de 2013.

12 La LIONDAU (2003) se inspira en los principios de vida independiente, normalización, accesibilidad univer sal, diseño para todos, diálogo civil y transversalidad de las políticas en materia de discapacidad. A estos efectos, entiende por: a) Vida independiente: la situación en la que la persona con discapacidad ejerce el poder de decisión sobre su propia existencia y participa activamente en la vida de su comunidad, conforme al derecho al libre desarrollo de la personalidad. b) Normalización: las personas con discapacidad deben poder llevar una vida normal, accediendo a los mismos lugares, ámbitos, bienes y servicios que están a disposición de cualquier otra persona. c) Accesibilidad universal: condición que deben cumplir los entornos, procesos, bienes, productos y servicios, así como los objetos o instrumentos, herramientas y dispositivos, para ser comprensibles, utilizables y practicables por todas las personas en condiciones de seguridad y comodidad y de la forma más autónoma y natural posible. d) Diseño para todos: la actividad por la que se concibe o proyecta, desde el origen, y siempre que ello sea posible, entornos, procesos, bienes, productos, servicios, objetos, instrumentos, dispositivos o herramientas, de tal forma que puedan ser utilizados por todas las personas, en la mayor extensión posible. e) Diálogo civil: el principio en virtud del cual las organizaciones representativas de personas con discapacidad y de sus familias participan, en los términos que establecen las leyes y demás disposiciones normativas, en la elaboración, ejecución, seguimiento y evaluación de las políticas oficiales que se desarrollan en la esfera de las personas con discapacidad. f) Transversalidad de las politicas de discapacidad: principio en virtud del cual las actuaciones que desarrollan las Administraciones públicas no se limitan únicamente a planes, programas y acciones específicos, pensados exclusivamente para estas personas, sino que comprenden las políticas y líneas de acción de carácter general en cualquiera de los ámbitos de actuación pública, en donde se tendrán en cuenta las necesidades y demandas de las personas con discapacidad.

13 El Movimiento de Vida Independiente aconseja usar el término de diversidad funcional en lugar de discapacidad. Se trata de substituir los conceptos peyorativos sobre las limitaciones por la visión positiva de la funcionalidad y dar voz a los implicados: Nada para las personas con discapacidad sin las personas con discapacidad (Declaración de Madrid, 2003).

Consultar: wmw.forovidaindependiente.org y wnw.derechoshumanosya.org.

El Guiniguada, No $22(2013) \cdot$ Las Palmas de Gran Canaria 


\section{Teresa Fontán Montesinos, Gisela Rivero Marrero}

Romper el ciclo de la invisibilidad. El empleo como vía para favorecer la inclusión

14 La a asociación SOLCOM se constituyó para la solidaridad comunitaria de las personas con diversidad funcional y la inclusión social. Entre sus actividades se encuentra: a) organizar grupos y redes de apoyo jurídico y multidisciplinares, b) formalizar peticiones, quejas, reclamaciones, recursos, demandas, denuncias, querellas o cualquier otra acción jurídica ante cualquier organismo, entidad, administración, juzgado, tribunal o corte, fuese de ámbito internacional, europeo, español, autonómico o local, y c) realizar estudios y proyectos en los campos jurídico, económico, social, asistencial, sanitario o en cualquier otra disciplina que resulte idónea de conformidad con los fines asociativos. Consultar http:/ / www. asociacionsolcom.org.

15 Consulte las publicaciones del Ministerio de Educación tituladas: Datos y cifras de la Educación en España, que se elaboran cada curso escolar, y compare esos datos.

16 Una de las primeras consecuencias de la entrada en vigor de la LOMCE, para el próximo curso 2014 2015, será la entrada en vigor de la Formación Profesional Básica y la supresión de los Programas de Cualificación Profesional Inicial (PCPI), el escalón más básico de la Formación Profesional, con la cualificación de nivel 1 del Catálogo Nacional de las Cualificaciones Profesionales, que tenían la gran ventaja de propiciar la obtención del Título de ESO al finalizar el segundo año de los módulos voluntarios. (...) la titulación, ha sido la principal motivación para el alumnado de PCPI para cursar estos estudios y ha sido, de hecho, una herramienta que ba permitido a miles de estudiantes, reengancharse al sistema educativo. Los PCPI han sido un instrumento eficaz.para asegurar la titulación de miles de alumnos que, de otra manera, estarian en la calle sin la titulación más básica en España. Con la entrada en vigor de la LOMCE se desvincula la Formación Profesional Básica con la obtención del Título de ESO. Por otra parte, es una incógnita qué sucederá con el alumnado con necesidades especificas de apoyo que actualmente pueden cursar los PCPI en las modalidades general y especial. http:/ / wnw. feccoocyl.es 